\title{
Co-design and robots: a case study of a robot dog for aging people
}

\author{
Tuck W Leong and Benjamin Johnston \\ Faculty of Engineering and IT \\ University of Technology Sydney \\ Broadway, NSW, Australia
}

\begin{abstract}
The day-to-day experiences of aging citizens differ significantly from young, technologically savvy engineers. Yet, well-meaning engineers continue to design technologies for aging citizens, informed by skewed stereotypes of aging without deep engagements from these users. This paper describes a co-design project based on the principles of Participatory Design that sought to provide aging people with the capacity to co-design technologies that suit their needs. The project combined the design intuitions of both participants and designers, on equal footing, to produce a companion robot in the form of a networked robotic dog. Besides evaluating a productive approach that empowers aging people in the process of co-designing and evaluating technologies for themselves, this paper presents a viable solution that is playful and meaningful to these elderly people; capable of enhancing their independence, social agency and well-being.
\end{abstract}

Keywords: Participatory Design, Co-design, Robot Dog, Aging

\section{Introduction}

A great achievement of many developed countries is that their citizens are now living longer. For example, it is estimated that by 2056, 25\% of Australia's population will be aged 65 years and above [3], approximately twice as many as the population in 2011. This brings huge challenges and opportunities in domains including healthcare, gerontology, social policy and technology design.

Technology designers, including those working with robots, have long engaged with efforts to understand and design technologies for aging people (e.g., $[1,2,4])$. However, some point out that these efforts are often skewed by dominant discourses surrounding aging and older people; stereotyping aging people negatively as a group who are needy, frail, lonely and have difficulties using new technologies $[6,12,14]$. Such a view misses the fact that many aging people are reasonably healthy, socially active, and would learn and use technologies if they believe that it is useful and relevant to their needs [17]. Instead of designing technologies that reflect aging people's values [13] we get a lot of technologies that are deficit driven (e.g., [5]) and assistive in nature (e.g., [17]). For example, 
when robots are considered, they are often viewed as a technological fix for the aging and yet older adults are often not consulted when designing robots [12].

To counter this, Vines et al. [22] recommend using a participatory process during design to produce technologies that fit well with aging people's aspirations. This provides people a voice throughout and allows them to decide the measure of success that is meaningful to them. Vines et al. [22] also recommend having deeper engagements with small groups of individuals to produce designs that respond to their personal histories and everyday lived experiences.

The design project described in this paper is a case study that exemplifies Vines et al.'s [22] recommendations, where designers worked collaboratively with a small group of older people to explore and co-design ideas and solutions that are meaningful to them. In particular, this paper demonstrates how this approach can be used productively and effectively with aging people to come up with the idea of, and to explore the use of, a robot to support and even enhance their day-to-day experiences of aging. The final design concept, a robot dog and associated use scenarios were developed, refined and evaluated iteratively with the participants. These outputs were further evaluated at a later stage by elderly participants at two different workshops. Overall, the feedback about the concept was very positive, demonstrating that aging people can accept the idea of a robot companion if it is perceived to be both playful and useful by them to achieve their desires to age well and live longer in their own homes.

\section{A participatory approach to technology design}

Following Vines et al.'s [22] recommendations, Participatory Design (PD) processes were used with a group of aging people. The process saw five designers working with eight elderly participants over interviews and three workshops. The designers consisted of four postgraduate Interaction Design students and an academic supervisor experienced in Interaction Design and Participatory Design. The participants were four males and four females in good health and aged 65-75. The participatory design process used in the project is depicted in Figure 1.

This PD process alternates between mutual learning activities (i.e., sessions with the participants) and refining activities (i.e., where designers conduct background research and develop prototypes). A novel aspect of this process is that it does not begin by teaching users about the capabilities/limitations of robotics (cf., $[12,19,1])$, yet incorporates the user in the earliest stages of design. This ensures a focus on user needs without being derailed by a focus on particular technologies. In fact, the designers approached the project agnostic about the technological solution; the idea of robotics as a potential solution was not in the designers' minds prior to, nor during the early parts of the design process.

Mutual learning is a core commitment of Participatory Design (PD) and is "one of its key differentiators from User-Centred Design and other humancentred approaches" [18]. This is because PD recognizes that people who are not professional technology designers cannot actively participate in co-designing 


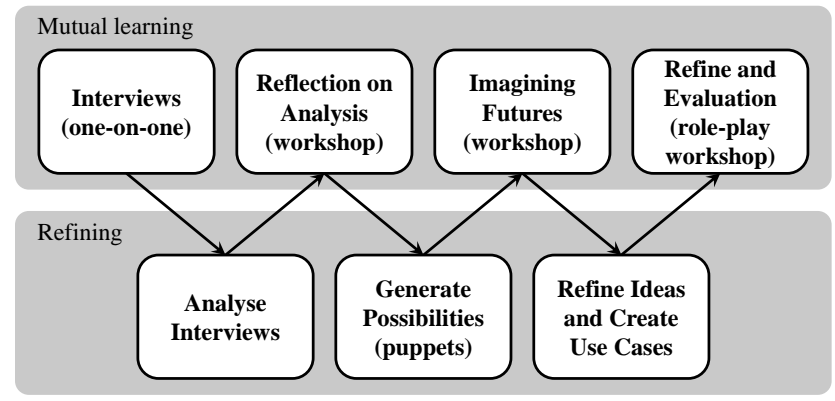

Fig. 1. Proposed co-design process

technologies they might use because they lack knowledge about what advanced technologies could offer them (e.g., [7,20]). Mutual learning is made possible through the initial face-to-face interviews and the three follow-up workshops. These activities allow designers to learn more about the participants and for participants to learn about possibilities of technologies, and together learn about possible and useful technology solutions.

The refining activities in the process are concerned with extending what designers learned about the users, ideation, research, developing prototypes of the users' ideas and preparing for the mutual learning workshops.

\section{$2.1 \quad$ Interviews}

Working in pairs, the designers conducted a series of one-to-one open-ended interviews with each participant at their homes. The interviews were audiorecorded and spanned approximately an hour each. The aim was to understand participants' experiences of aging, e.g. what is important to them as they age, their situations, life histories, hopes and fears, use of technologies. All audiorecordings were transcribed, and the data was analysed using thematic analysis.

Analysis of the interviews revealed the busy lives participants kept, with various activities such as gardening, home projects, socializing with friends or family members. Their engagement with life was to keep fit physically, mentally and emotionally. One of the main reasons for this was their desire to remain independent for as long as possible and continue to live in their homes as they age. Participants who were single did speak about occasionally feeling lonely. This made them anxious and sad, something they really wish to avoid.

Two participants own a dog at home and extolled its benefits. For them, the dog was a source of comfort and companionship. Dogs can also warn them of strangers approaching their homes, giving them a sense of security, especially for those living alone. Walking their dogs regularly helped them stay healthy and also provide opportunities to socialize with other dog walkers. 
While all participants own a smartphone, all of them use a limited number of apps. The phone is used primarily as a communication tool with friends and family. Finally, all of the participants lamented on their growing forgetfulness and absentmindedness, especially with their phones. They often forget to charge their phones (resulting in missed communications) or sometimes are not able to find their phones (resulting in frustration).

\subsection{Workshop 1 (Reflection and Validation of Analysis)}

Workshop 1 followed the interviews. The aim is to provide the participants an opportunity to learn about and discuss the interview findings.

The workshop began with a presentation of selected commercial/consumer technologies that have been designed for aging citizens. Participants were encouraged to ask questions and to discuss how these technologies could potentially affect their lives. After this, participants were presented with the findings from the interviews. They were given the opportunity to discuss the findings and seek further clarifications. Besides providing some validation of the designers' analysis, the activity allowed participants to provide further insights and clarifications to some of the findings. Having the opportunity to listen to each other's experiences of aging also spurred further discussions and ideas.

One very popular discussion was centered around the benefits of having a pet dog at home, how the dog may support their aspirations to age well and in their own homes and the challenges of finding housing suitable for dog ownership. By the end of Workshop 1, the participants were asked to vote on what they wanted to co-design in further workshops. They chose to focus on finding ways to feel safe, calm, and connected while at home but at the same time like to discover ways to expand their social circles.

\subsection{Generating Possibilities}

Given the significant interests and discussions surrounding the benefits dogs, and a realisation that a 'dog' might be an interesting design idea, the designers decided to explore the idea of a robot dog in the second workshop.

Using robots to assist and support older adults to maintain their independence, and enhance their well-being is not a new idea in Human-Robot Interaction (e.g., $[1,19])$. As highlighted earlier, the application (and design) of technologies, including robots are often driven by (erroneous) assumptions about aging people or by goals to reduce human carers. Beer et al.'s [4] review of domestic robots efforts highlight various ways robots have been proposed to help with aging people, e.g., performing home upkeep tasks, especially for individuals with declined health capabilities, such as motor impairments, dementia, or needing healthcare assistance. Kumahara and Mori [11] developed a mobile robot that can walk together with elderly people and help them to maintain their healthy condition. Robotic dogs have also been proposed, with efforts to improve its emotional recognition capabilities [9] to make it more engaging. Others explored children's reaction to robot dogs when compared to a real one [15]. While the 
range of efforts are impressive, they are still focused upon on assistive features for sick or needy individuals, forgetting that a large number of aging individuals, are still healthy, active, and capable of doing things for themselves.

Meanwhile, a lot of work with robots in the domestic setting have focused on whether they are accepted by people, and ways to better design for this acceptance (e.g. $[8,16,23])$. Most 'design work' with domestic robots begins with presenting people with various robots to evaluate, such as the kind of appearances and functionalities that are important to older adults. These efforts often do not consider aging people's emotional needs and experiences when living with these robots, such as companionship, security and even day-to-day 'social' interactions with these robots. An exception is the recent work by Lazar et al. [12] that used focus groups with older adults to explore some of the situatedness of aging. While they offer some insights and directions for future design, they did not fully develop the ideas nor provided concrete use scenarios.

In this project, the designers took the findings from the interviews and Workshop 1, and conducted background research about robot use in aging, especially within domestic settings. In addition, the designers needed to find ways to stimulate the participants' imagination of a future scenario with a robot in their lives in the second workshop. After some speculation about possible designs, the Sony AIBO (a dog-like entertainment robot capable of locomotion, sensing and communication by sound and WiFi) was selected as the 'puppet' to be used to support participants imagination and an inoperable AIBO was sourced.

\subsection{Workshop 2 (Imagining Futures)}

While the interviews and Workshop 1 were focussed on learning about users' situations and needs. The aim of the second workshop was to use the same participants to explore and co-design the idea of a robot dog.

As explained earlier, participants would need some understanding about technologies if they were to participate effectively in co-design activities. Thus, the second workshop began with presentations from the designers regarding domestic robots and emergent technologies such as the Internet of Things. Participants learned about the kinds of technologies available and the potential of a networked environment. When the participants encountered examples whereby robots are used to support aging people, such as acting as 'carers', all of them found the idea very inappropriate and demeaning - "not something I could see myself wanting, no thanks, I am not that useless, yet". They asked questions and sought clarifications about various technologies presented but always in reference to their own situations and needs.

After the presentations, an imaginative activity with the participants were conducted where the participants were introduced to the AIBO puppet (hereby known as the dog). The designers had attached sticks and strings to the dog and one of the designers acted as the 'puppeteer', making decisions as to what the dog might respond to in this workshop.

The participants were told that their task is to imagine what the dog could do for them in their everyday lives. They were fascinated by it and at first, the 
participants interacted with the dog as they would with a real dog: asking it to come, to sit, to fetch things and so on. Since the dog did not always respond as asked, it was interesting to see how forgiving people were. Then, one of the designers hinted to the participants of the possible properties of some emergent technologies, such as connectivity.

After some silence, one participant asked, "Can it connect to my phone and find it? I always misplace it and I can't find it when I need it." When the designers confirmed that the dog could be tethered to their phones, the participants suddenly became very excited. They had so many questions. "Will it tell me if my phone is ringing? Sometimes I can't hear it ring". "What about when my battery is low?" "What about when the phone is silent?', etc. The designers did their best to provide reasonably realistic answers and at the same time encouraged the collective exploration of ideas. At first, everyone was excited about the number of things the dog could do but one of the participants indicated that she really prefers "a companion, not an assistive device... something that dogs do but just a bit more because it is special and hardy". Throughout, two designers captured the participant's ideas. Before the workshop ended, the participants ranked the ideas and worked with the designers to write out scenarios for their top three ideas.

\subsection{Design work informed by Workshop 2}

After Workshop 2, three detailed and rich scenarios for 'Hardy Hound' (the name for this robot dog) were refined by the designers for presentation in the final third workshop. The scenarios were based closely on the participants' ideas. Below is a brief description of the three scenarios.

Activity 1: Finding a phone. When users cannot find their mobile phone, they can ask the dog to look for it because the dog is tethered to the phone. The dog will lead the user to the phone.

Activity 2: Increasing social interaction. A Hardy Hound is designed to recognise other Hardy Hounds. When it locates another dog close by, it will try and seek out the other dog. The dogs' interaction provides a 'ticket to talk': taking a Hardy Hound for a walk may provide users with chances of communicating with other Hardy Hound users, thus, the potential to make friends and increase their level of social interaction.

Activity 3: Companionship. When the user is feeling lonely, she can call out to the Hardy Hound. The dog will go to the owner and behave in a happy manner.

Lucy Suchman's interaction framework [21] was chosen to be used in Workshop 3 to refine the basic interactions between people and the dog. Suchman pointed out that human-computer communication is a special case of communication whereby the resources available to the participants is limited, and how this could lead to problems and breakdowns. In the case of a robotic dog, its capacity to interact is limited by its programming, only able to respond to specific commands (input) as deemed plausible, given current technology. 


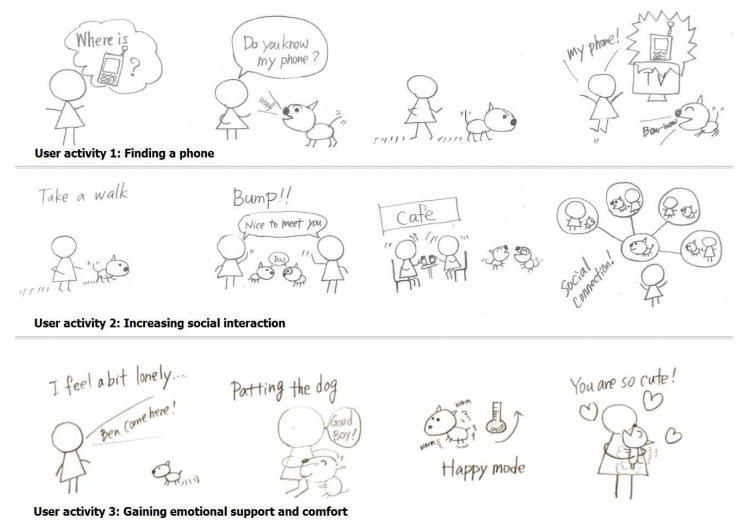

Fig. 2. Three scenarios for Hardy Hounds

\subsection{Workshop 3 (Refine and Evaluation)}

Workshop 3 aimed to learn some of the potential communicative problems and to prototype the necessary interactions. The workshop was driven by role play: one of the designers put on a dog suit and mask, acting as a 'Ben, a Hardy Hound'. He was given his 'programming' for each activity which determines (and limits) his capacity to act/behave when interacting with the participants. The participants were introduced to Ben and were briefed about Scenario 1. They were told that Ben has limited programming and would only understand some verbal commands. They then took turns interacting with Ben to find a phone. This was then repeated for Scenario 2 and 3. The activities were videotaped. In between each activity, discussions were held to elicit participants' experiences, expectations and thoughts.

Problems arose immediately because people were issuing different kinds of commands. For example, to find a phone, they asked "Where's my phone?", "Phone", and "Get my phone", instead of the "programmed' command of "Find my phone". Because participants needed to be told of the 'correct' command before using using it, the importance of more clear user feedback became apparent, e.g., using audio and visual feedback such as barking and moving forward. The richness of the feedback provided thorough insights into the important features of human-machine intelligibility.

The outcome of the final workshop was a more refined set of interactions for each of the scenarios. This also included recommendations, such as enabling users to customize commands and the need for consistency of feedback from the dog. The scenarios were also refined. It was obvious from the session that users wanted the dog to behave and respond as similarly as possible to a real dog, e.g., not using human speech but barks, body language, eyes, ears, tail. More interestingly, the design team found that people were very forgiving of the dog. If the dog did not respond as they expected, they just laughed it off and attempted 
different ways to interact with it. Instead of being frustrated, they found it cute. Even when the dog's responses are ambiguous, people were more than willing to interpret and try to make sense of what they believe the dog is "trying to say".

\section{Further Evaluation}

Six months after the original design workshops, the scenarios and interactions were evaluated at two additional workshops with new groups of aging people.

Each workshop consisted of eight people (16 total) aged 65-90 to explore values that aging people hold dear as they age. Understanding aging people's values can help shed light on why aging people adopt (or not) technologies such as our robot dog [13].

During the workshop, various technologies were presented to elderly participants to evaluate based upon their values. The Hardy Hound concept and its scenarios were presented as one such technology. Participants were asked to evaluate how well the concept fit with their needs, situations and values as well as any other uses they could they imagine.

The results were very positive. Most of them (14/16) liked the technology, seeing it as "cute", a "good companion" and "helping to keep my blood pressure down". None imagined that it would replace a real dog but it was certainly a viable alternative for apartment dwellers or nursing homes where live pets were not allowed. In fact, two participants were worried that their real-life dogs might "destroy the robot". 4/16 wanted to know where they could get one right away. While the functionalities were desirable, they also saw it as a machine for play and companionship. In fact, a few thought that owning such a dog would give them a certain amount of cachet or admiration from their peers and more importantly, their grandchildren. Many of them could also imagine different ways whereby the Hardy Hounds might be useful in their everyday lives, such as to strengthen a sense of security when they are home alone.

\section{Discussion}

This paper makes the following contributions to the robotics community. First, a participatory approach that supports elderly people to imagine creative applications of technologies for themselves. It demonstrates the capacity of elderly people to act as active partners in co-design - able to envisage and articulate solutions they will use, that are suitable and meaningful to their needs and aspirations. This approach stands in contrast with most approaches where aging people are generally not consulted. While there aren't many examples of PD in robotics, some, such as Šabanović et al. [19] have used PD to design socially assistive robots with older adults. This paper provides another example of how PD can be used, but emphasising the value of alternating phases of mutual learning and reflection/refining in this PD process.

Second, it presents a set of use scenarios and learnings about interactions for a robot dog that have been developed with and evaluated by elderly people. 
The concept is well received by the participants invovled and is ready for further prototyping and development. This work concurs with recent findings by Lazar et al. [12], such as the capacity of a robot pet to enhance social interactions with others. As such, this work provides a strong foundation for an approach and a viable concept related to a robot dog. We have not reported the lengthy set of interactions but instead highlight the usefulness of Suchman's framework [21] to help decompose, understand, analyse the real and complex problem of designing people's interactions with robots.

Third, this work demonstrates that participants did not find the idea of interacting and living with a robotic dog to be problematic. This contrasts with others working in Human-Robot interactions (e.g., [10, 23]), who found barriers to robot-acceptance, including older adults' uneasiness with technology, feeling of stigmatization, and ethical/societal issues associated with robot use. We would argue that the co-design process that was used helped mitigate many of these concerns.

As the world's aging population grows, efforts to design interactive technologies will need to reconsider their approach. We have reported one way where designers can engage productively with older adults directly in the design and evaluation of technology. With planning and support, aging people are more than capable of imagining and co-designing technologies that not only meet their needs but fit their values and can co-exist with them meaningfully in their everyday lives.

\section{References}

1. Alves-Oliveira, P., Petisca, S., Correia, F., Maia, N. and Paiva, A., 2015, October. Social robots for older adults: Framework of activities for aging in place with robots. In International Conference on Social Robotics, pp. 11-20.

2. Ahn, H.S., Kuo, I.H., Datta, C., Stafford, R., Kerse, N., Peri, K., Broadbent, E. and MacDonald, B.A., 2014, October. Design of a Kiosk Type Healthcare Robot System for Older People in Private and Public Places. In International Conference on Simulation, Modeling, and Programming for Autonomous Robots, pp. 578-589.

3. Australian Bureau of Statistics. 2009. Future Population Growth and Aging.

4. Beer, J.M., Smarr, C-A., Chen, T.L., Akanksha, P., Mitzner, T.L., Kemp, C.C., and Rogers, W.A. 2012. The domesticated robot: design guidelines for assisting older adults to age in place. In Proceedings of Human Robot Interactions (HRI'12), pp. 335-342.

5. Carroll, J., Convertino, G., Farooq, U. and Rosson, M.B. 2012. The firekeepers: Aging considered as a resource. The Information Society, 11 (1), pp. 7-15.

6. Durick, J., Robertson, T., Brereton, M., Vetere., F and Nansen, B. 2013. Dispelling aging myths in technology design. In Proceedings of the 25th Australian Computer-Human Interaction Conference (OzCHI'13), pp. 467-476.

7. Greenbaum, J. and Kyng, M., 1991. Design at work: Cooperative design of computer systems. Lawrence Erlbaum Associates.

8. Heelink, M. 2011. Exploring the influence of age, gender, education and computer experience on robot acceptance by older adults. In Proceedings of the 6th international conference on Human-robot interaction (HRI'11), pp. 147-148 
9. Jones, C.M. and Demming, A. 2007. Investigating emotional interaction with a robotic dog. In Proceedings of the 19th Australasian conference on ComputerHuman Interaction (OzCHI'07) pp. 183-186.

10. Khosla, R., Chu, M-T., Kachouie, R., Yamada, K. and Yamaguchi, T. 2012. Embodying care in Matilda - an affective communication robot for the elderly in Australia. In Proceedings of of the 2nd ACM SIGHIT International Health Informatics (IHI'12), pp. 295-304.

11. Kumahara, Y and More, Y. 2014. Portable robot inspiring walking in elderly people. In The Second International Conference on Human-Agent Interaction (HAI 2014), pp. 145-148.

12. Lazar, A., Thompson, H.J., Piper, A.M. and Demiris, G. 2016. Rethinking the Design of Robotic Pets for Older Adults. In Proceedings of the 2016 ACM Conference on Designing Interactive Systems, pp. 1034-1046.

13. Leong, T.W. and Robertson, T. 2016. Voicing values: laying foundations for aging people to participate in design. In Proceedings of the 14th Participatory Design Conference.

14. Light, A., Leong, T.W., and Robertson, T. 2015. Aging well with CSCW. In Proceedings of the 14th European Conference on Computer Supported Cooperative Work (ECSCW 2015), pp. 295-304

15. Melson, G. Khan, P.H., Beck, A.M., Friedman, B., Roberts, T. and Garrett, E. 2005. Robots as dogs? Children's interaction with the robotic dog AIBO and a live Australian Shepherd. In Proceedings of CHI '05 EA Extended Abstracts on Human Factors in Computing Systems, pp. 1649-1652.

16. Prakash, A, Kemp, C.C. and Rogers, W.A. 2014. Older adults' reactions to a robot's appearance in the context of home use. In Proceedings of the 2014 ACM/IEEE international conference on Human-robot interaction.

17. Robertson, T., Durick, J., Brereton, M., Vetere, F., Howard, S. and Nansen, B. 2012. Knowing Our Users: Scoping Interviews in Design Research with Aging Participants. In Proceedings of the 24th Australian Computer-Human Interaction Conference, pp, 517-520.

18. Robertson, T., Leong, T.W., Durick, J., and Koreshoff, T. 2014. Mutual learning as a resource for research design. In Proceedings of the 13th Participatory Design Conference (PDC'14), pp. 25-28.

19. Šabanović, S., Chang, W.L., Bennett, C.C., Piatt, J.A. and Hakken, D., 2015, August. A robot of my own: participatory design of socially assistive robots for independently living older adults diagnosed with depression. In International Conference on Human Aspects of IT for the Aged Population, pp. 104-114.

20. Simonsen, J. and Robertson, T. (Eds.) 2012. Routledge International Handbook of Participatory Design. Routledge.

21. Suchman, L. 1987. Plans and situated actions: The problem of Human-Machine Communication. Cambridge University Press, New York.

22. Vines, J. Pritchard, G., Wright, P., Olivier, P. and Brittain, K. 2015. An Age Old Problem: Examining the discourses of aging in HCI and strategies for future research. In ACM Transactions on Computer-Human Interaction (TOCHI), 22(1), Article 2.

23. Wu, Y-H., Wrobel, J., Cornuet, M., Damne., and Rigaud, A-S. 2014. Acceptance of an assistive robot in older adults: a mixed-method study of humanrobot interaction over a 1-month period in the Living Lab setting. Clinical Interventions in Aging, 14(9), pp. 801-811. 